\section{IN BRIEF}

\section{RHEUMATOID ARTHRITIS}

\section{ACPA-negative RA divided into clinical subsets}

The multi-biomarker disease activity (MBDA) score, an algorithm based on 12 serological markers, helped subdivide patients with anti-citrullinated protein antibody (ACPA)-negative rheumatoid arthritis (RA) into clinically relevant subsets in a multivariable analysis. Patients with a high or medium MBDA score at the time of diagnosis had a higher likelihood of sustained DMARD-free remission than patients with a low score $(P=0.115)$. This association was independent of clinical baseline characteristics and driven primarily by levels of $C$-reactive protein, serum amyloid $A$ and matrix metalloproteinase 3

ORIGINAL ARTICLE Boeters, D. M. et al. ACPA-negative RA consists of subgroups: patients with high likelihood of achieving sustained DMARD-free remission can be identified by serological markers at disease presentation. Arthritis Res. Ther. 21, 121 (2019)

\section{CONNECTIVE TISSUE DISEASES}

\section{Pregnancy outcomes in patients with MCTD}

In a multicentre retrospective analysis of 94 ever-pregnant women with mixed connective tissue disease (MCTD) who tested positive for anti-U1RNP antibodies, 147 of the 203 pregnancies $(72.4 \%)$ resulted in live births, 38 (18.7\%) ended in a first trimester pregnancy loss and 18 (8.9\%) ended in a stillbirth. The fetal outcomes were worse for women who also tested positive for antiphospholipid antibodies or had pulmonary or muscular involvement. Specific counselling was advised for patients with MCTD who are planning a pregnancy.

ORIGINAL ARTICLE Radin, M. et al. Pregnancy outcomes in mixed connective tissue disease: a multicentre study. Rheumatology. https://doi.org/10.1093/rheumatology/ kez141 (2019)

\section{SYSTEMIC SCLEROSIS}

\section{Nintedanib slows ILD progression in SSc}

Treatment with nintedanib, a tyrosine kinase inhibitor, slowed the progression of interstitial lung disease (ILD) associated with systemic sclerosis (SSc) in a 52-week randomized placebocontrolled trial. The adjusted annual rate of decline in forced vital capacity was lower in the nintedanib-treated group than in the placebo-treated group $(P=0.04)$, although no clinical benefits for other manifestations of SSc were observed. The adverse-event profile was similar to that reported in previous trials of patients with idiopathic pulmonary fibrosis; the rate of gastrointestinal adverse events, including diarrhoea, was higher in the nintedanib group than in the placebo group.

ORIGINAL ARTICLE Distler, O. et al. Nintedanib for systemic sclerosis-associated interstitial lung disease. N. Engl.J. Med. https://doi.org/10.1056/NEJMoa1903076 (2019)

\section{RHEUMATOID ARTHRITIS}

\section{Hyperuricaemia or gout in patients with RA}

In a large longitudinal study of 1,999 patients with rheumatoid arthritis (RA) in the USA, the prevalence of gout (6.1\%) and hyperuricaemia (17\%) was similar to previous estimates of these conditions in the general population. The coexistence of hyperuricaemia or gout in patients with RA was not associated with RA disease activity or severity. However, moderate hyperuricaemia in these patients was strongly associated with an increased risk of cardiovascular-diseaserelated mortality, which was driven by the presence of other comorbidities.

ORIGINAL ARTICLE Chiou, A. et al. Coexistent hyperuricemia and gout in rheumatoid arthritis: associations with comorbidities, disease activity and mortality. Arthritis Care Res. https://doi.org/10.1002/acr.23926(2019)

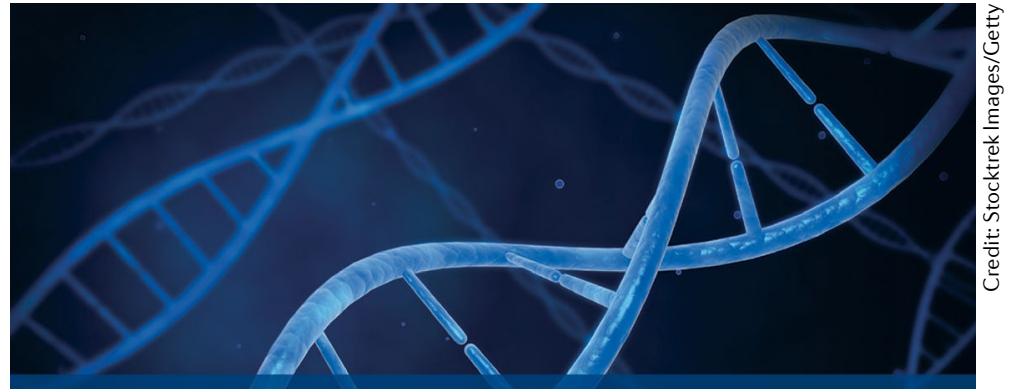

$\Rightarrow$ SYSTEMIC LUPUS ERYTHEMATOSUS

\title{
Rare variants in SLE risk genes drive disease
}

New research reveals that most patients with systemic lupus erythematosus (SLE) carry rare, coding variants in genes implicated in SLE risk; moreover, these variants have damaging effects on protein function and ultimately contribute to the development of autoimmunity. Together, the findings of the study by Simon Jiang and colleagues suggest that rare and novel variants are important in the pathogenesis of SLE.

To date, the prevailing view has been that genetic predisposition to SLE arises from the additive effect of many common variants that have been identified over the past few decades using genome-wide association studies and that individually have modest effect sizes. However, emerging evidence has indicated that rare variants with strong effect sizes contribute to SLE in some individuals.

In an initial investigation in two cohorts of patients with SLE (133 patients in total) and 97 healthy elderly individuals, Jiang et al. found that $82 \%$ of patients with SLE and $72 \%$ of healthy individuals carried at least one rare nonsynonymous single-nucleotide variant (SNV) in SLE-associated genes. In particular, a substantial proportion of patients with SLE carried rare or low-frequency variants in $B L K$ and/or BANK1.

Further studies demonstrated that the SNVs in BLK (encoding tyrosine-protein kinase BLK) identified in patients with SLE altered the kinase activity of BLK, resulting in impaired phosphorylation of interferon regulatory factor 5 (IRF5) and B-cell scaffold protein with ankyrin repeats (BANK1). SLE-associated BLK variants also failed to repress IRF5-mediated type I interferon expression in comparison with wild-type BLK.

Jiang et al. also demonstrated that a BANK1 SNV promotes type I interferon activity owing to impaired sequestration of TNF receptor-associated factor 6 (TRAF6), which was associated with increased TRAF6-mediated nuclear localization of IRF5.

"We have shown for the first time that rare variants in SLE-associated genes, specifically $B L K$ and $B A N K 1$, have deleterious effects on their translated proteins," says Jiang. "Interestingly, rare variants in these genes in healthy individuals do not affect protein function."

In lupus-prone Fas ${ }^{l p r / p r}$ mice, introduction of a Blk variant orthologous to one found in SLE led to expansion of pathogenic T cells, demonstrating a contribution to the development of disease in vivo.

The researchers plan to elucidate the disease-related mechanisms of other rare variants in SLE-associated genes and potential insights into new targets for treatment, such as exploring anti-interferon therapy for patients bearing mutations associated with increased type I interferon activity. "By identifying the genetic mechanisms which contribute to SLE, we may be able to stratify patients further by their genetic variants as well as trial treatments targeted for specific disrupted pathogenic pathways," concludes Jiang.

Sarah Onuora

ORIGINAL ARTICLE Jiang, S. H. et al. Functional rare and low frequency variants in BLK and BANK1 contribute to human lupus. Nat. Commun. 10 , 2201 (2019) 